\title{
Mapping the Effects of Three Dopamine Agonists with Different Dyskinetogenic Potential and Receptor Selectivity Using Pharmacological Functional Magnetic Resonance Imaging
}

\author{
Marina Delfino',2, Raffael Kalisch², Michael Czisch², Celia Larramendy', Jimena Ricatti', Irene RE Taravini', \\ Claudia Trenkwalder' ${ }^{2}$, Mario Gustavo Murer*,3, Dorothee P Auer ${ }^{2}$ and Oscar S Gershanik' \\ 'Laboratorio de Parkinsonismo Experimental, Instituto de Investigaciones Farmacológicas, CONICET, Buenos Aires, Argentina; ${ }^{2}$ Max-Planck- \\ Institute of Psychiatry, Munich, Germany; ${ }^{3}$ Laboratorio de Fisiología de Circuitos Neuronales, Departamento de Fisiología y Biofísica, Facultad \\ de Medicina, Universidad de Buenos Aires, Buenos Aires, Argentina
}

\begin{abstract}
The mechanisms underlying dopamine agonist-induced dyskinesia in Parkinson's disease remain poorly understood. Similar to patients, rats with severe nigrostriatal degeneration induced by 6-hydroxydopamine are more likely to show dyskinesia during chronic treatment with unselective dopamine receptor agonists than with D2 agonists, suggesting that DI receptor stimulation alone or in conjunction with D2 receptor stimulation increases the chances of experiencing dyskinesia. As a first step towards disclosing drug-induced brain activation in dyskinesia, we examined the effects of dopamine agonists on behavior and blood oxygenation level-dependent (BOLD) signal in the striatum and motor cortex of rats with unilateral nigrostriatal lesions. Rats were rendered dyskinetic before pharmacologic functional magnetic resonance imaging by means of a repeated treatment regime with dopamine agonists. The unselective agonist apomorphine and the selective DI/D5 agonist SKF-8I297 induced strong forelimb dyskinesia (FD) and axial dystonia and increased BOLD signal in the denervated striatum. Besides, SKF-81297 produced a significant but smaller BOLD increase in the intact striatum and a symmetric bilateral increase in the motor cortex. The D2 family agonist quinpirole, which induced mild dyskinesia on chronic treatment, did not produce BOLD changes in the striatum or motor cortex. Further evidence to support an association between BOLD changes and dyskinesia comes from a direct correlation between scores of FD and magnitude of drug-induced BOLD increases in the denervated striatum and motor cortex. Our results suggest that striatal and cortical activation induced by stimulation of DI/D5 receptors has a primary role in the induction of peak dose dyskinesia in parkinsonism.

Neuropsychopharmacology (2007) 32, 191 I-1921; doi:I0.1038/sj.npp. I 301329; published online 7 February 2007
\end{abstract}

Keywords: Parkinson's disease; dyskinesia; dopamine agonist; BOLD; pharmacological MRI

\section{INTRODUCTION}

Parkinson's disease (PD) is caused by a progressive degeneration of nigrostriatal dopaminergic pathways. Chronic administration of drugs that stimulate striatal dopamine receptors produce symptomatic relief in this condition. However, disabling motor complications, notably dyskinesia, develop in as much as $50 \%$ of patients treated for 5 years or longer (Obeso et al, 2000). Yet, the mechanisms underlying dopamine agonist-induced dyskinesia remain poorly understood.

\footnotetext{
*Correspondence: Dr MG Murer, Laboratorio de Fisiología de Circuitos Neuronales, Departamento de Fisiología y Biofísica, Facultad de Medicina, Universidad de Buenos Aires, Paraguay 2155, Buenos Aires ( I I I I), Argentina, Tel: 54 | | 59509500 ext. 2137, Fax: 54 I I 4963 8593, E-mail: park-exp@ffyb.uba.ar

Received 3 August 2006; revised 28 November 2006; accepted II December 2006
}

In patients, the severity of nigrostriatal degeneration, the duration of antiparkinsonian drug therapy and the pharmacokinetics and pharmacodynamics of antiparkinsonian drugs influence the probability of developing dopamine agonist-induced dyskinesia (Nutt, 2000). On repeated administration, unselective dopamine agonists with short half-lives in plasma are more likely to induce dyskinesia than D2 receptor agonists with long half-lives in plasma (Nutt, 2000). Less is known about the dyskinetogenic potential of D1/D5 receptor agonists; yet, it seems that they are able to induce dyskinesia in patients (Rascol et al, 2001).

Recent findings show that rats with unilateral nigrostriatal degeneration induced by 6-hydroxydopamine (6OHDA) exhibit spontaneous motor deficits and dopamine agonist-induced abnormal involuntary movements (AIMs) that share functional similarities with $\mathrm{PD}$ akinesia and dopamine agonist-induced dyskinesia (Cenci et al, 2002). In rodents, the susceptibility to develop dyskinesia is related to the extent of dopamine axon terminal loss in the striatum 
and to the nature of drug treatment in a way that closely resembles the situation in patients. Repeated exposure to dopamine agonists increases the likelihood of developing dyskinesia, and unselective dopamine agonists (apomorphine, levodopa) are more likely to induce dyskinesia than selective D2 family receptor agonists (bromocriptine, quinpirole) in severely lesioned rats (Lundblad et al, 2002; Winkler et al, 2002; Delfino et al, 2004). Taken together, data from patients and animal models suggest that sensitization of striatal neurons to D1 and/or combined D1 and D2 receptor stimulation contribute to drug-induced dyskinesia.

Monitoring brain activity changes induced by selective and unselective dopamine agonists in rodent models of PD might help gain insight into the mechanisms underlying dopamine agonist-induced dyskinesia. Functional magnetic resonance imaging (fMRI) allows for indirect assessment of the level of neuronal activity by measuring concomitant changes in blood oxygenation. The so-called blood oxygenation level-dependent (BOLD) signal can be used not only to study activity changes induced by sensory or cognitive stimuli but also changes induced by drugs, a variant that is termed pharmacological fMRI (phMRI). Several studies have been able to demonstrate BOLD signal increases following administration of non-selective dopamine agonists in animal models of PD (Chen et al, 1997, 1999; Nguyen et al, 2000; Kalisch et al, 2005). The value of this technique is demonstrated by the contrasting effects that apomorphine and the dopamine releasing compound amphetamine have on behavior and basal ganglia signal in rats with unilateral nigrostriatal lesions induced by 6-OHDA. Apomorphine induces contralateral turning behavior (an index of striatal denervation supersensitivity) and accordingly increases the BOLD signal on the lesioned side, whereas amphetamine produces ipsilateral turning (related to the extent of nigrostriatal damage) and increases BOLD on the intact side (Ungerstedt, 1976; Chen et al, 1997; Nguyen et al, 2000). This consistency between lateralized BOLD signal changes and well-characterized neurophysiological effects motivated us to compare, by means of phMRI, different selective and non-selective dopamine agonists in rats with unilateral nigrostriatal damage. We aimed to assess their acute effects on striatal and motor cortex activation as well as the effect of repeated preexposure to dopamine agonists on acute dopamine agonistinduced activation changes in these rats. This was accompanied by quantification of drug-induced dyskinetic behavior. We predicted that the non-selective agonist apomorphine and the D1/D5 selective agonist SKF-81297 would induce stronger activation in (ipsilateral) striatum and motor cortex as well as stronger dyskinesia than the D2 selective agonist quinpirole upon acute administration. We further predicted that pre-exposure would enhance these effects. Confirmation of these predictions would establish a direct link between the behavioral phenomenon of dyskinesia and a pharmacological response of the motor system.

\section{MATERIALS AND METHODS}

Forty-two male Wistar rats $(298 \pm 41 \mathrm{~g})$ purchased from Charles River (Germany) were used for the experiments. They were kept in groups of three per cage in a temperature-controlled room $\left(21^{\circ} \mathrm{C}\right)$ with a $12: 12 \mathrm{~h}$ lightdark cycle (lights on at 0700 hours) and ad libitum access to food pellets and tap water.

\section{6-OHDA Lesion}

All rats received a unilateral 6-OHDA injection in the medial forebrain bundle, which was performed following a published protocol (Murer et al, 1998). Briefly, rats were pretreated with desipramine $(25 \mathrm{mg} / \mathrm{kg}$ i.p.) $30-45 \mathrm{~min}$ before stereotaxic lesioning, to protect norepinephrine nerve terminals. Under deep surgical inhalatory anesthesia (isoflurane) each rat received an injection of 6-OHDA $(3.75 \mathrm{mg} / \mathrm{ml}, 4 \mu \mathrm{l} / \mathrm{min}$, in order to produce a severe lesion) into the left medial forebrain bundle (stereotaxic coordinates: $2.8 \mathrm{~mm}$ caudal to bregma; $2.0 \mathrm{~mm}$ from midline; $8.6 \mathrm{~mm}$ ventral from brain surface; tooth bar: $-3.3 \mathrm{~mm}$ below the interaural line) (Paxinos and Watson, 1986).

\section{Experimental Design}

As our main aim was to compare the ability of different dopamine agonists to induce dyskinesia and BOLD changes in the striatum and motor cortex, it was important to ascertain by functional means that rats were severely lesioned and uniformly supersensitive at the beginning of the study. In 6-OHDA-lesioned rats, depletion of dopamine neurons both in the substantia nigra pars compacta and the ventral tegmental area, as well as striatal dopamine receptor supersensitivity, correlate with the degree of contraversive turning after an apomorphine challenge (Ungerstedt, 1976; Murer et al, 1998; Nguyen et al, 2000). Therefore, all rats were challenged with apomorphine $(0.25 \mathrm{mg} / \mathrm{kg}$, s.c. $) 2$ weeks after surgery (Figure 1a) and contraversive turning was measured. The extent of 6-OHDA-induced damage was further studied by post-mortem immunohistochemistry to detect tyrosine hydroxylase (TH) in striatal sections.

Six weeks after surgery (Figure 1a), the animals were randomly assigned to one of the following treatments: apomorphine (unselective agonist), quinpirole (D2 family selective agonist), SKF-81297 (D1/D5 selective agonist), or vehicle. Each treatment group was further divided into two subgroups. Subgroup 1 received a dopamine agonist challenge every 3 days for 18 days (seven injections). Behavioral evaluation of AIMs was performed after the first and seventh drug challenges. This repeated pre-exposure schedule maximizes behavioral sensitization to drugs (Morelli et al, 1989; see also Delfino et al, 2004). An eighth drug challenge was then given 3 days after finishing the sensitization protocol, whereas BOLD was measured using phMRI (groups apomorphine-eighth, $n=6$; quinpiroleeighth, $n=6$; SKF-81297-eighth, $n=6$; vehicle-eighth $n=3$ ). Subgroup 2 received only a single drug challenge, during phMRI, and no behavioral evaluation of AIMs was performed (groups apomorphine-first, $n=6$; quinpirolefirst, $n=6$; SKF-81297-first, $n=6$; and vehicle-first, $n=3$ ).

The doses used for the behavioral experiments (apomorphine $0.25 \mathrm{mg} / \mathrm{kg}$, quinpirole $0.1 \mathrm{mg} / \mathrm{kg}$, SKF-81297 $0.1 \mathrm{mg} /$ $\mathrm{kg}$, all i.p.) were found in pilot studies to induce comparable rates of contraversive turning behavior (data not shown; see also Delfino et al, 2004). Dosage was balanced according to rotational behavior because contraversive turning is an 
a

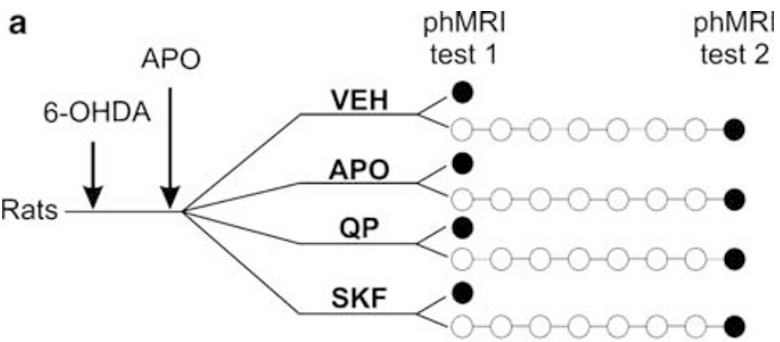

drug challenge in awake animals

drug challenge inside scanner
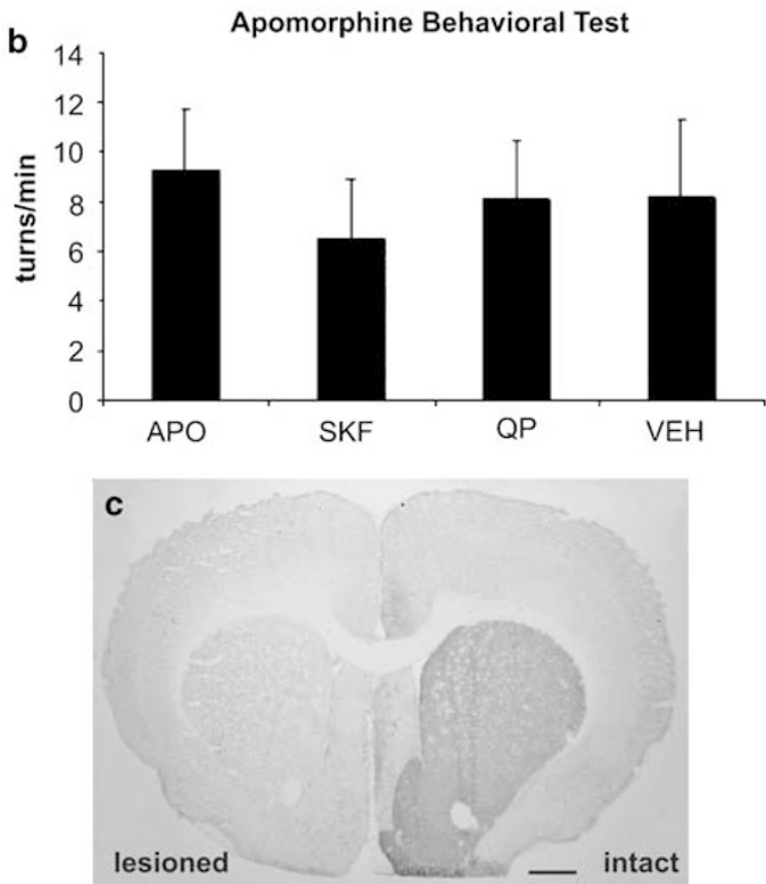

Figure I (a) Schematic illustration of the experimental design (see text for details). (b) Two weeks after 6-OHDA administration, all rats were assessed for apomorphine (Apo)-induced contraversive turning behavior. Rats showing more than five net contraversive turns per min were randomly assigned to receive a dopamine receptor agonist or vehicle. The resulting four groups showed similar net contraversive turning rates after apomorphine (one-way ANOVA). (c) Post-mortem immunohistochemistry revealed a severe depletion of $\mathrm{TH}$ immunoreactive nerve terminals in the striatum in all animals used for the experiments. The picture shows a representative example. Scale bar: I mm. Veh: vehicle; SKF: SKF-8 I297; QP: quinpirole; 6-OHDA: 6-hydroxydopamine.

index of severe nigrostriatal damage and postsynaptic supersensitivity (Ungerstedt, 1976), which in turn have been linked to dyskinesia (Nutt, 2000). Antiparkinsonian effects can be obtained with lower doses of dopamine agonists (Olsson et al, 1995) and there is no correlation between contraversive rotational behavior and improvement in motor disability (Lundblad et al, 2002; Metz and Whishaw, 2002). Therefore, the drug-induced changes in BOLD described here can be related to dyskinesia. However, it is less clear whether they are related to antiparkinsonian efficacy or not. As most general anesthetics, including isoflurane, diminish neuronal activity, and BOLD responses (for a discussion on this topic see the review by Steward et al, 2005), we increased the respective doses to $1.25,0.5$, and $0.5 \mathrm{mg} / \mathrm{kg}$ (i.p.) for the phMRI sessions. Usage of higher drug doses than in behavioral experiments is in line with earlier studies from our group (Kalisch et al, 2005) and other groups (eg, Chen et al, 1997).

\section{Behavioral Testing}

Apomorphine-induced turning behavior was evaluated 2 weeks after surgery in a circular arena $30 \mathrm{~cm}$ in diameter. Full turns were quantified every minute between minutes 5 and 20 after injection. The rats selected for this study displayed mean net contraversive turning rates above 5 per min (Figure 1b).

AIMs (Cenci et al, 1998; Lundblad et al, 2002; Delfino et al, 2004) were evaluated after the first and seventh drug injections of the sensitization schedule, during 2 min every $30 \mathrm{~min}$, for $2 \mathrm{~h}$ post-injection. Forelimb dyskinesia (FD), axial dystonia $(\mathrm{AD})$ and masticatory dyskinesia $(\mathrm{MD})$ were scored separately. Scores go from 0 to 4 (0: no dyskinesia; 1 , occasional; 2 , frequent; 3 , continuous, interrupted by sensory distraction; 4, continuous, not interrupted by sensory distraction). Behavioral tests were performed between 1100 and 1700 hours.

\section{phMRI Methodology}

Animals were prepared as described previously (Kalisch et al, 2001). Briefly, they were orally intubated, mechanically ventilated under $1.5 \%$ isoflurane and catheterized (tail artery, tail vein, i.p., s.c.). Pancuronium (Curamed, Germany) was administered i.v. as needed for muscular relaxation but was never given during phMRI scanning to avoid related blood pressure (BP) modulations. Body temperature and expiratory $\mathrm{CO}_{2}$ were continuously monitored and kept constant throughout the experiment. BP was also monitored continuously via the tail artery. Druginduced BP changes can generate correlated BOLD signal changes, which may mask neurally mediated drug effects on BOLD (Kalisch et al, 2001; Nagaoka et al, 2002; Tuor et al, 2002; Kalisch et al, 2005; Wang et al, 2006). In order to minimize such BP changes, all animals received domperidone (0.4 mg i.v.; Motilin, BYK, Germany), an antagonist of peripheral D2 family receptors, $10 \mathrm{~min}$ before scanning. Furthermore, we used a recently established BP clamping technique (Kalisch et al, 2005) by which BP is kept constant with the help of a continuous i.v. infusion of the adrenergic agonist phenylephrine (PEP; $10 \mu \mathrm{g} / \mathrm{ml}$ in phosphate-buffered saline (PBS); Sigma, Germany). In this method, PEP infusion volumes are continuously adjusted by the experimenter whenever BP begins to deviate from the baseline level. The method has been shown by us to reduce BP confounds and to improve the detectability of non-BPmediated drug-induced BOLD signal changes (Kalisch et al, 2005).

Scans were performed on a Bruker 7T Avance Biospec 70/30 MRI magnet (Bruker, Germany) with a receive-only saddle-shaped surface coil specially designed for rat brain. For the functional scans, a T2-sensitive rapid acquisitionrelaxation enhanced sequence was used: $\mathrm{TE}_{\text {eff. }}=19.5 \mathrm{~ms}$, $\mathrm{TR}=1875 \mathrm{~ms}$, echo train length: 6 , slice thickness: $1 \mathrm{~mm}$, FOV: $3.5 \times 3.5 \mathrm{~cm}^{2}$, acquisition matrix: $96 \times 96$, image size $128 \times 128,0.273 \times 0.273 \mathrm{~mm}^{2}$, temporal resolution: 2 
volumes per $\mathrm{min}, 180$ repetitions $(90 \mathrm{~min}), 32$ dummy excitations ( $1 \mathrm{~min})$. To achieve interindividually comparable striatal scans, a package of 13 axial slices of $1 \mathrm{~mm}$ thickness was positioned between the most posterior point of the olfactory bulb as the anterior and the base of the fourth ventricle as the posterior border of the slice package. Inter-slice gaps were adapted individually for each animal to fit the package into these borders, thus accounting for differences in brain size. This way, slices 3 and 4 reproducibly cover the anterior and central part of the striatum, approximately corresponding to Paxinos and Watson's Figures 11-18, and slice 2 covers a rostral and medial part of the motor cortex, corresponding to Figures 8-10 (Paxinos and Watson, 1986).

Dummy Scans were acquired before baseline in order to reach signal stability. Apomorphine (Teclapharm, Germany), quinpirole (Sigma), SKF-81297 (Sigma), or $0.5 \mathrm{ml} / \mathrm{kg}$ vehicle were injected $15 \mathrm{~min}$ after starting image acquisition at a rate of $0.4 \mathrm{ml} / \mathrm{min}$ through an i.p. plastic catheter. Image acquisition continued for $75 \mathrm{~min}$ postinjection.

\section{Immunohistochemistry}

At the end of experiment, anesthesia was deepened and the animal decapitated. Brains were removed, frozen in isopentane at $-30^{\circ} \mathrm{C}$ and stored at $-80^{\circ} \mathrm{C}$ until processing. The extent of nigrostriatal damage was estimated by means of $\mathrm{TH}$ immunohistochemistry performed on striatal sections. Coronal, $20-\mu \mathrm{m}$-thick tissue sections were cut at $-20^{\circ} \mathrm{C}$ in a cryostat and mounted on gelatin-coated glass slides. After washing with PBS $(0.1 \mathrm{M})$, tissue sections were treated in a blocking solution of $0.1 \mathrm{M}$ PBS containing $2 \%$ BSA and $0.3 \%$ Triton X-100 (30 min) and incubated overnight at $4{ }^{\circ} \mathrm{C}$ with polyclonal rabbit anti-TH antibodies (P-40101, Pel Freez, Rogers, Arkansas) (working dilution 1:500). Three rinses in PBS were performed before incubating the sections with anti-rabbit IgG (Vector Laboratories) (working dilution 1:250). The antibodyantigen complex was visualized by means of an avidinbiotin peroxidase complex (ABC Elite, Vector Laboratories) and developed with 3,3'-diaminobenzidine and $\mathrm{H}_{2} \mathrm{O}_{2}$ in Tris Buffer $0.1 \mathrm{M}(0.1 \% \mathrm{w} / \mathrm{v}$ and $0.015 \% \mathrm{v} / \mathrm{v}$, respectively).

\section{Data Analysis and Statistics}

Regions of interest (ROIs) in left and right striatum and motor cortex were predefined based on comparison with the atlas of Paxinos and Watson (1986), using a circular (striatum) and an ellipsoid (motor cortex) template (see Figure $3 \mathrm{a} 1$ and a2). Voxel-wise raw BOLD time courses were averaged over these ROIs and drug- or vehicle-induced BOLD signal change was expressed as percentage of the baseline activity during the $15 \mathrm{~min}$ preceding drug challenge. No spatial or temporal data smoothing was performed. In some experiments, we observed a progressive linear decrease of the BOLD signal starting approximately 45 post-injection. Such drifts are sometimes observed during long scans (see, eg, Kalisch et al, 2005) but their causes are unclear. As we had no functional hypotheses regarding these drifts, data analysis was restricted to the first $45 \mathrm{~min}$ post-injection.
ANOVA was used for statistical comparisons followed by post hoc Least Significant Difference (LSD) tests (Statistica, StatSoft Inc., Tulsa, OK, USA). Pearson correlations were calculated between BOLD signal and behavioral data.

\section{RESULTS}

Two weeks after 6-OHDA administration, rats were tested for apomorphine-induced turning behavior and then randomly assigned to a drug treatment or vehicle (Figure 1a). The resulting four animal groups displayed similar and marked apomorphine-induced contraversive turning (Figure 1b) at rates that were compatible with severe degeneration of the mesotelencephalic dopaminergic pathways (Ungerstedt, 1976). Post-mortem immunohistochemistry confirmed the existence of an almost complete depletion of $\mathrm{TH}+$ axon terminals in the striatum and a severe reduction in nucleus accumbens, on the side ipsilateral to the 6-OHDA injection (Figure 1c). This is in agreement with previous own results showing $>90 \%$ reduction of immunoreactivity for $\mathrm{TH}$, dopamine transporter and vesicular monoamine transporter type II in the striatum in the same rat 6-OHDA model (Salin et al, 1997; Murer et al, 1998).

\section{AIMs Induced by Dopamine Receptor Agonists}

To assess the behavioral effects of various dopamine agonists and their modulation by repeated pre-exposure, half of the rats (subgroup 1) were then subjected to an 18-day sensitization schedule with either apomorphine, quinpirole, SKF-81297, or vehicle, and AIMs were measured after the first and seventh drug challenges (Figure 1a). Previous reports indicated that the non-selective agonist apomorphine is a more potent inductor of AIMs than D2 family agonists (Lundblad et al, 2002; Delfino et al, 2004). In good agreement with these findings, apomorphine induced strong $\mathrm{FD}$ and $\mathrm{AD}$, whereas the $\mathrm{D} 2$ agonist quinpirole produced a lower number of AIMs (Figure 2). It should be noticed that FD and $\mathrm{AD}$ are abnormal movements, so observers hardly ever score one such movement in vehicle controls (Cenci et al, 1998; Lundblad et al, 2002). Overall, these findings confirm earlier reports by our group (Delfino et al, 2004) showing that quinpirole reliably induced AIMs in rats, which have been primed with apomorphine. Interestingly, and in analogy to the effects of a D1/D5 selective agonist in patients (Rascol et al, 2001), the D1/D5 selective agonist SKF-81297 also induced marked FD and AD. For MD, only SKF-81297 differed significantly from the other treatments $(p=0.0005 v s$ apomorphine; $p=0.03$ vs quinpirole; $p=0.004 v s$ vehicle). Note that, in contrast to what happens with $\mathrm{AD}$ and $\mathrm{FD}$, normal mouth movements are difficult to discriminate from $\mathrm{MD}$, explaining the nonzero MD scores of the vehicle-treated rats. This suggests that rodent $\mathrm{MD}$ could be a less reliable measure of druginduced dyskinesia than FD and AD.

Figure 2 shows that FD and AD scores were slightly higher at the seventh than at the first drug challenge for all drug treatments, yet, the sensitization factor only showed trendlevel significance, indicating that only weak or no further sensitization occurred after the first drug challenge, 

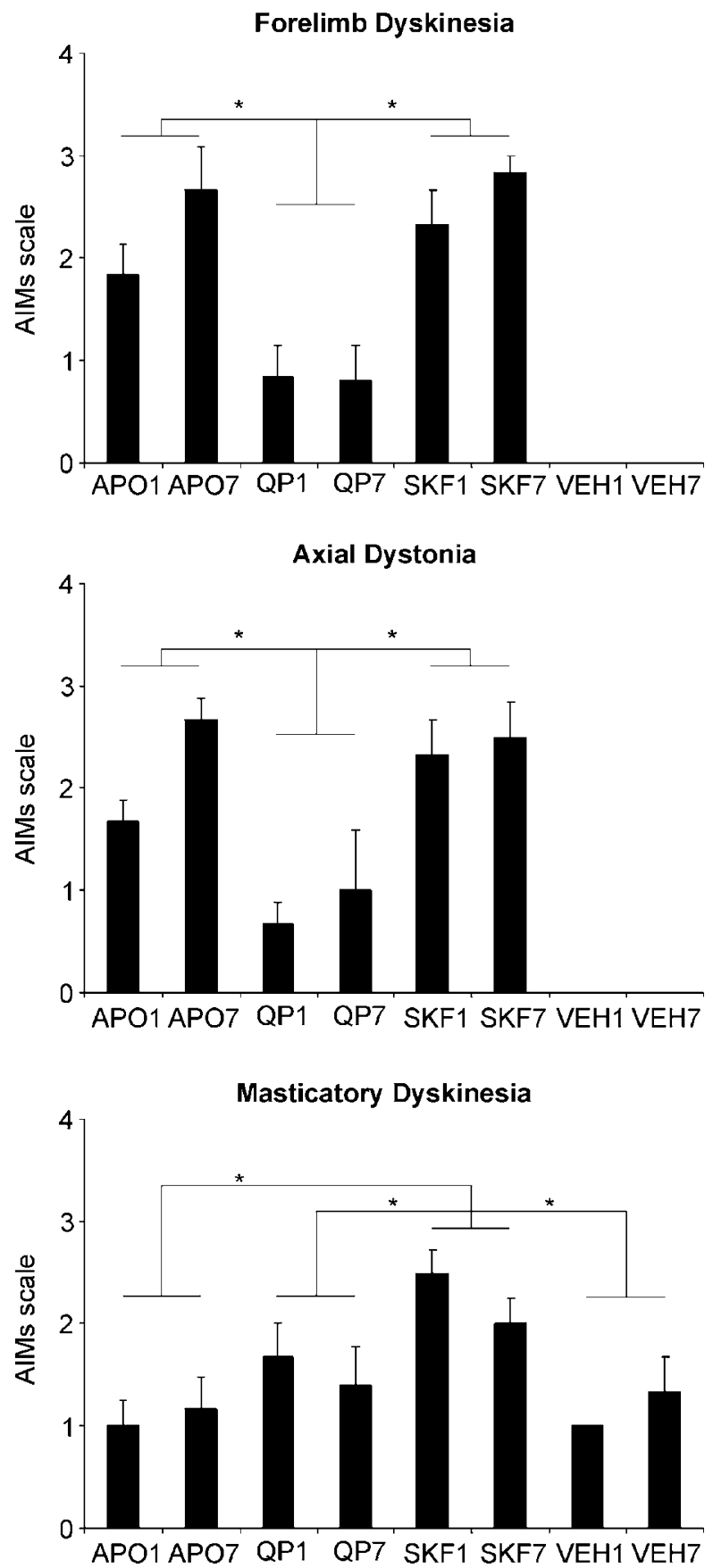

Figure 2 AIM scores attained the first and seventh drug or vehicle challenge by the animals subjected to a dopamine-agonist sensitization schedule (subgroup I). The vehicle-treated rats scored zero FD and AD. Two-way ANOVAs with drug treatment (apomorphine, quinpirole, SKF81297, vehicle) and sensitization (first vs seventh challenge) as betweenand within-subject factors, respectively, showed significant effects of drug treatment for the three types of abnormal movement (FD: $F_{(2,14)}=10.48$, $\left.p=0.0016 ; A D: F_{(2,14)}=10.53, p=0.0016 ; M D: F_{(3,16)}=7.24, p=0.0027\right)$. The sensitization factor missed significance $\left(F D: F_{(1,14)}=4.51, p=0.052\right.$; AD: $\left.F_{(1,14)}=4.02, p=0.06 ; M D: F_{(I, 16)}=0.18, p=0.67\right)$ and there was no interaction. Apomorphine (APO) and SKF-81297 (SKF) induced significantly more FD and AD than quinpirole (QP) (*p $<0.05$, LSD test). Regarding MD, apomorphine and quinpirole did not differ from vehicle, and SKF-8I 397 differed significantly from all other treatments $(* p<0.05$, LSD test). $\mathrm{VEH}$ : vehicle. possibly because of the apomorphine challenge the rats had received 2 weeks after 6-OHDA lesioning (Delfino et al, 2004). As FD and AD did not attain maximum scores at the seventh drug challenge, it seems unlikely that a ceiling effect could have masked the development of behavioral sensitization during repeated drug testing.

\section{BOLD Signal Changes Induced by Dopamine Receptor Agonists}

We examined the effects of dopamine agonists on BOLD in the two subgroups of 6-OHDA-lesioned rats (Figure 1a): the above subgroup 1 , in which rats had received seven challenges before being scanned during an eighth challenge, and which could therefore be assessed for AIMs in addition to BOLD (see above; treatment groups apomorphine-eighth, quinpirole-eighth, SKF-81297-eighth and vehicle-eighth); and subgroup 2 in which rats were not previously exposed to drugs (with the exception of the apomorphine challenge 2 weeks after surgery) but received an agonist challenge during phMRI (treatment groups apomorphine-first, quinpirole-first, SKF-81297-first and vehicle-first). The analysis was limited to ROIs in (left and right) striatum and motor cortex for which average time courses were extracted from the images. BOLD signal changes were expressed as percent change relative to the 15 -min baseline before drug administration. Throughout the phMRI experiments, BP was controlled with the help of the phenylephrine BP clamping technique (Kalisch et al, 2005). BP did not differ from baseline in any experimental group (nonsignificant outcome of $t$-tests for paired data; Figure $3 \mathrm{a} 1$ and a2) and was similar in the different animal groups, as assessed by a two-way ANOVA with drug treatment $\left(\mathrm{F}_{(3,34)}=0.38\right.$; $p=0.76)$ and sensitization $\left(\mathrm{F}_{(1,34)}=3.13 ; p=0.09\right)$ as between-subject factors (interaction: $\mathrm{F}_{(3,34)}=2.25 ; p=0.1$ ).

Visual inspection of BOLD signal time courses in the preexposed subgroup 1 suggested that both apomorphine and SKF-81297 produced BOLD signal increases, mainly in striatum but also in motor cortex. BOLD signal increases induced by apomorphine were of moderate intensity and restricted to the ipsilateral side. SKF-81297 produced a marked bilateral BOLD signal increase both in striatum and motor cortex, although with preponderance in the side ipsilateral to the lesion (Figures 3 and 4). By contrast, in subgroup 2, which had not received pre-exposure, only SKF-81297 induced a noticeable BOLD signal change that appeared stronger in the striatum of the lesioned side.

Statistical analysis largely confirmed these results (Figure 4). Average BOLD signal changes from injection to minute 45 , relative to baseline, were subjected to threeway ANOVA with the two between-subject factors drug treatment (apomorphine, SKF-81297, quinpirole, or vehicle) and sensitization (ie, subgroup $1 v s$ subgroup 2) and the within-subject factor hemisphere. The analysis was carried out separately for striatum and motor cortex. In striatum (Figure 4a1), the three main factors (drug treatment: $\mathrm{F}_{(3,34)}=11.46 ; \quad p=0.0002 ; \quad$ sensitization: $\quad \mathrm{F}_{(1,34)}=4.6$; $p=0.04$; hemisphere: $\left.\mathrm{F}_{(1,34)}=17.04 ; p=0.0002\right)$ and the interaction between drug treatment and hemisphere $\left(\mathrm{F}_{(3,34)}=5.86 ; p=0.002\right)$ were all significant. SKF-81297 had a strong bilateral effect, that was significantly higher than that of any other treatment $(p=0.004 v s$ vehicle; 
a1
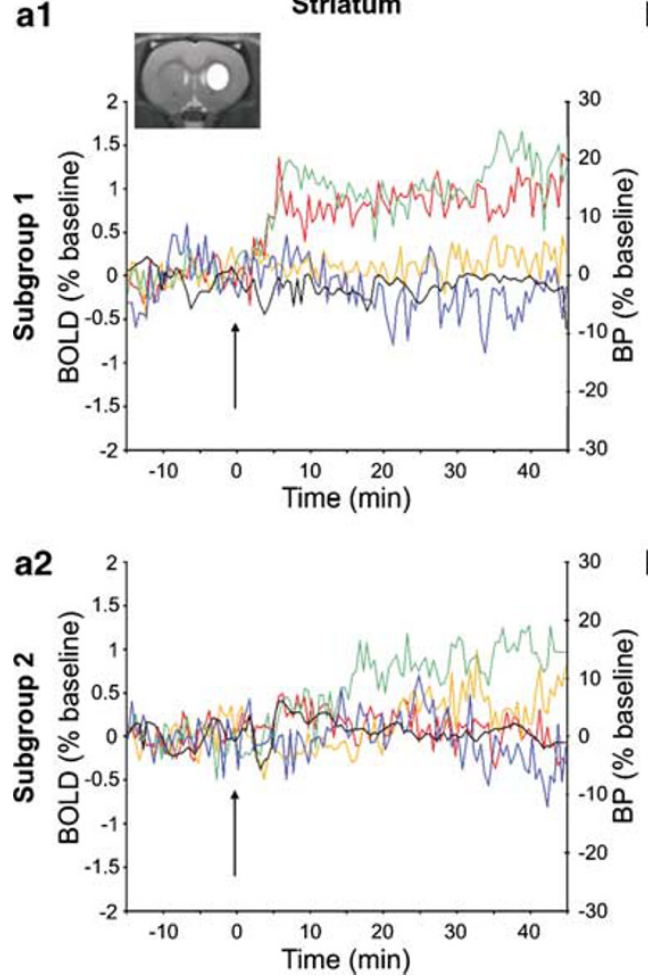

b1
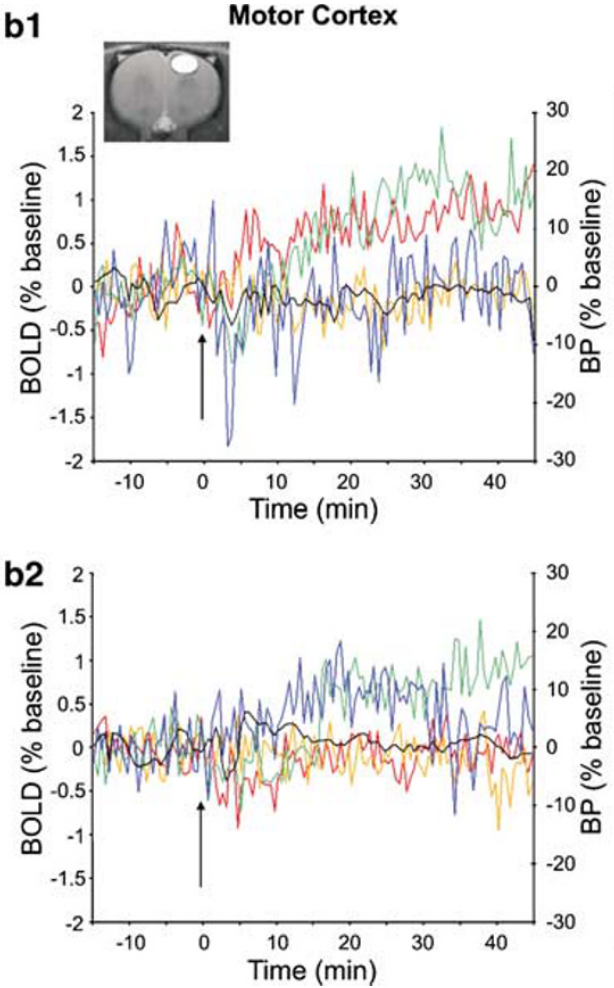

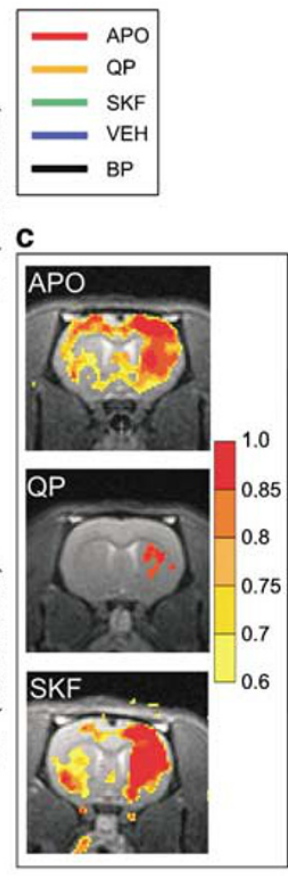

Figure 3 (a) Time course of average BOLD signal (ipsilateral minus contralateral values) in the striatum (a) and motor cortex (b), for subgroup I (sensitized, a l, bl) and subgroup 2 (non-sensitized, a2, b2). Insets: coronal images of the rat brain displaying the ROI from which BOLD signal was extracted. (c) Representative animals of each group are shown. A threshold at $>0.6$ was used for cross-correlation maps of BOLD signal changes and regional striatal time curves.

$p=0.0002$ vs apomorphine; $p=0.00009$ vs quinpirole; post hoc LSD tests), and significantly higher on the side ipsilateral to the lesion $(p<0.0001)$. The moderate apomorphine-induced increase in BOLD signal was not significantly different from quinpirole or vehicle effects. However, post hoc comparisons revealed a significantly higher effect of apomorphine on the striatum ipsilateral to the lesion ( $p=0.003$ compared to the contralateral striatum).

In motor cortex (Figure 4b1), there were also significant effects of drug treatment $\left(\mathrm{F}_{(3,34)}=7.64 ; p=0.0005\right)$ and sensitization $\left(\mathrm{F}_{(1,34)}=8.1 ; p=0.007\right)$ but not of hemisphere $\left(\mathrm{F}_{(1,34)}=1.83 ; p=0.18\right)$. A drug by sensitization interaction was also significant $\left(\mathrm{F}_{(3,34)}=3.71 ; p=0.02\right)$. Post hoc comparisons showed that SKF-81297 produced a significant bilateral BOLD signal increase in the pre-exposed subgroup 1 that was stronger than any other treatment in either subgroup $(p<0.001)$.

As the strong bilateral BOLD response induced by SKF81297 could have masked subtle effects of other drug treatments, we performed further analyses on the calculated difference between the ipsi- and contralateral BOLD signal by means of two-way ANOVA with treatment and sensitization as between-subject factors (Figure 4). It is believed that an inter-hemispheric difference in BOLD response of 6-OHDA-lesioned rats is correlated to striatal denervation supersensitivity (Nguyen et al, 2000) and therefore can be relevant to understand the mechanisms underlying dyskinesia. This analysis revealed asymmetric BOLD responses to SKF-81297 and apomorphine in striatum (Figure 4a2; drug treatment main effect:
$\mathrm{F}_{(3,34)}=5.86 ; p<0.0024 ;$ no significant effect of sensitization and no interaction; post hoc comparisons, $p=0.002$ SKF$81297 v s$ vehicle, $p=0.034$ apomorphine vs vehicle). No effects were observed in motor cortex (Figure 4b2).

To summarize, the phMRI results showed a higher sensitivity to dopaminergic stimulation with the nonselective agonist apomorphine and the D1/D5 selective agonist SKF-81297. Pre-exposure increased dopamine agonist-induced brain activation, both in the striatum and in the motor cortex. Also, the results seem to suggest a higher sensitivity to dopamine agonists of the striatum relative to the motor cortex. However, because neurovascular coupling and the sensitivity of the employed head coil may vary between brain areas, we refrained from any formal statistical comparison between the two regions.

\section{Correlation between BOLD Signal Changes and Behavior}

The above results would be further corroborated by correlations between dyskinesia scores and BOLD signal changes (Figure 5). As there were no significant BOLD signal changes in subgroup 2 (first challenge in phMRI), we restricted the analysis to a comparison of dyskinesia scores from the seventh challenge in subgroup 1 ( 3 days before phMRI) with BOLD signal changes at the eighth challenge in the same subgroup. In contrast to $\mathrm{AD}$ and $\mathrm{FD}$ scores, $\mathrm{MD}$ scores were not included in the analysis because they had shown only limited value as a measure of dyskinesia in our hands (see above). 

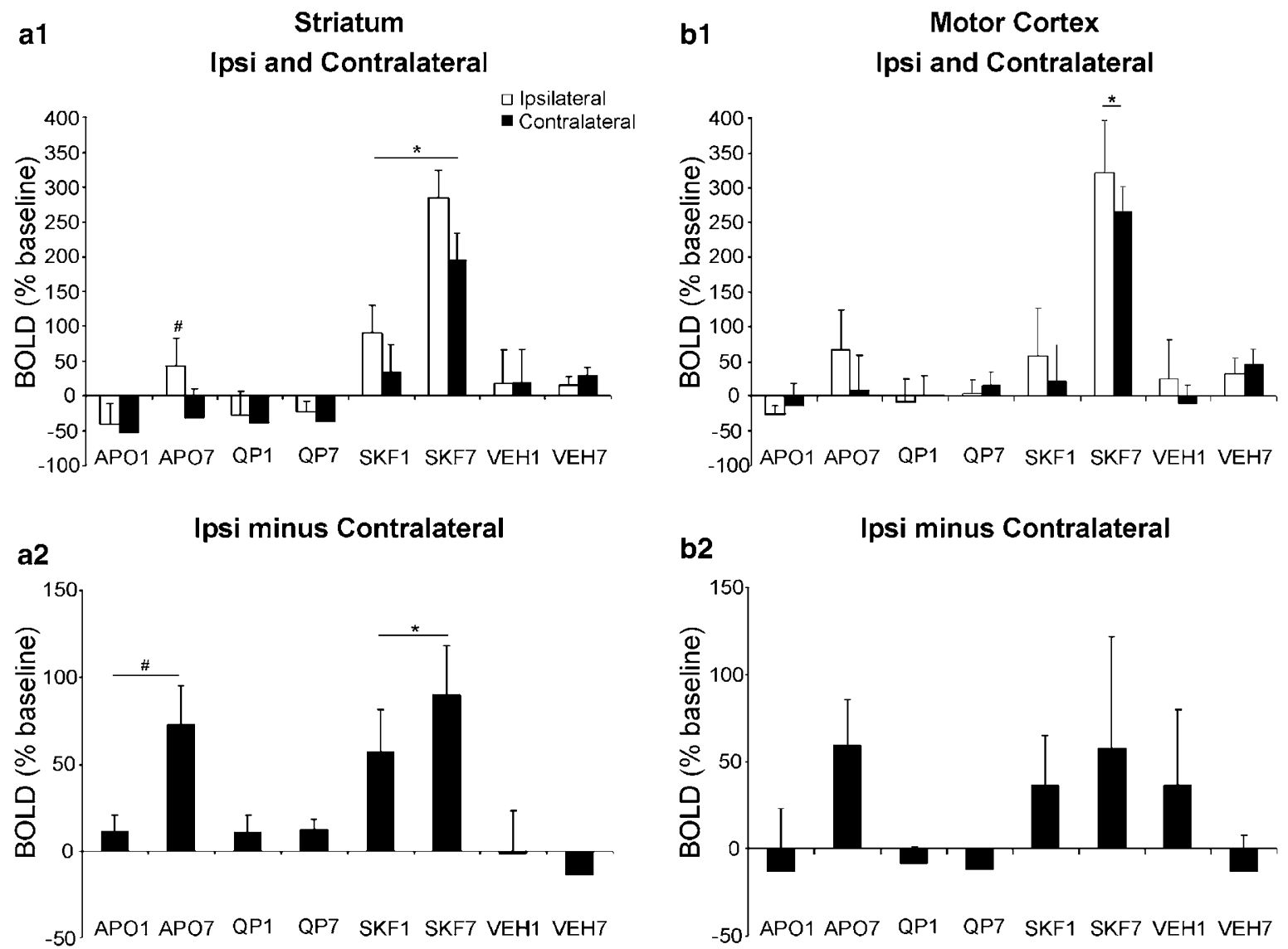

Figure 4 BOLD measures in the striatum (a) and motor cortex (b) across the 45 min post-injection period as percent of baseline, depicted separately for each brain side (al, bl) or as interhemispheric differences (a2, b2). al: * $p<0.01$ post hoc LSD test vs any other treatment; ${ }^{\#} p=0.003$ vs contralateral side within apomorphine. a2: ${ }^{*} p=0.002$ vs vehicle; ${ }^{\#} p=0.034$ vs vehicle. $b 1: * 0<0.00$ I vs any other treatment.

In contrast to our predictions, $\mathrm{AD}$ scores did not correlate with either ipsilateral or contralateral BOLD signal changes in either striatum or motor cortex. However, FD scores correlated significantly with BOLD increases in the denervated striatum $(r=0.52, p=0.02)$ and ipsilateral motor cortex $(r=0.53, p<0.016)$, but not with contralateral changes. The correlations with BOLD signal changes in the lesioned side were significantly higher than the correlations with contralateral changes. Given the small number of animals included in this study, the presence of significant and lateralized correlations with one of two employed behavioral measures can be regarded as a further corroboration of our results.

\section{DISCUSSION}

The effects of different dopamine agonists on behavior and on BOLD contrast in the striatum and motor cortex were analyzed in rats having chronic unilateral nigrostriatal lesions. On repeated administration, the non-selective dopamine agonist apomorphine and the D1/D5 agonist SKF-81297 induced strong dyskinesia of the trunk and forelimb contralateral to the lesion and increased BOLD in the ipsilateral striatum, whereas the selective D2 family agonist quinpirole, which induced mild AIMs, did not produce significant striatal BOLD changes. We further showed that there is a direct correlation between the magnitude of striatal and motor cortex BOLD responses and the severity of contralateral abnormal forelimb movements evoked by the drugs.

\section{D1/D5 Dopamine Receptor Agonists are More Powerful Inductors of AIMs Than D2 Family Agonists in Parkinsonian Rats}

Susceptibility of 6-OHDA-lesioned rats to develop AIMs is linked to the severity of nigrostriatal damage and the pharmacodynamics and pharmacokinetics of dopamine agonists (Cenci et al, 2002). In rats with severe nigrostriatal damage non-selective dopamine agonists are powerful inductors of AIMs. In contrast, the D2 family agonist quinpirole induce AIMs only if rats are first 'primed' with apomorphine (Delfino et al, 2004). Our present findings confirm that D1/D5 agonists can induce AIMs in parkinsonian rodents (Monville et al, 2005). Studies performed in MPTP-lesioned monkeys are to some extent contradictory on this matter, yet most emphasize that D1/D5 agonists induce significant dyskinesia (Blanchet et al, 1996; Pearce et al, 1999; Goulet and Madras, 2000) and the D1/D5 agonist ABT-431 is as potent as levodopa in inducing dyskinesia in patients (Rascol et al, 2001). Remarkably, we found that SKF-81297 induced significantly more MD than apomorphine and quinpirole, what is consistent with 


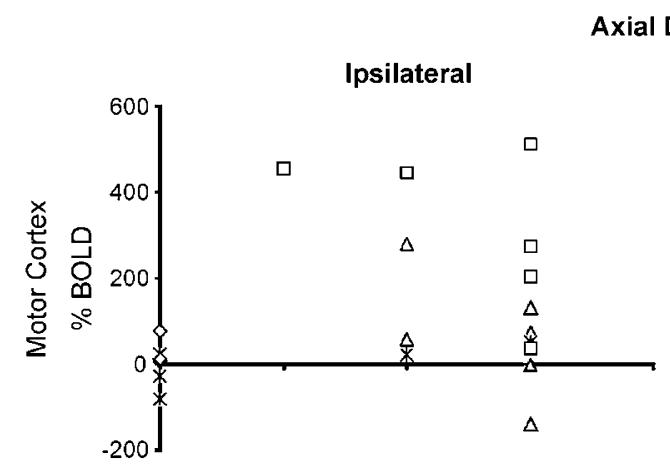

\section{Axial Dystonia}
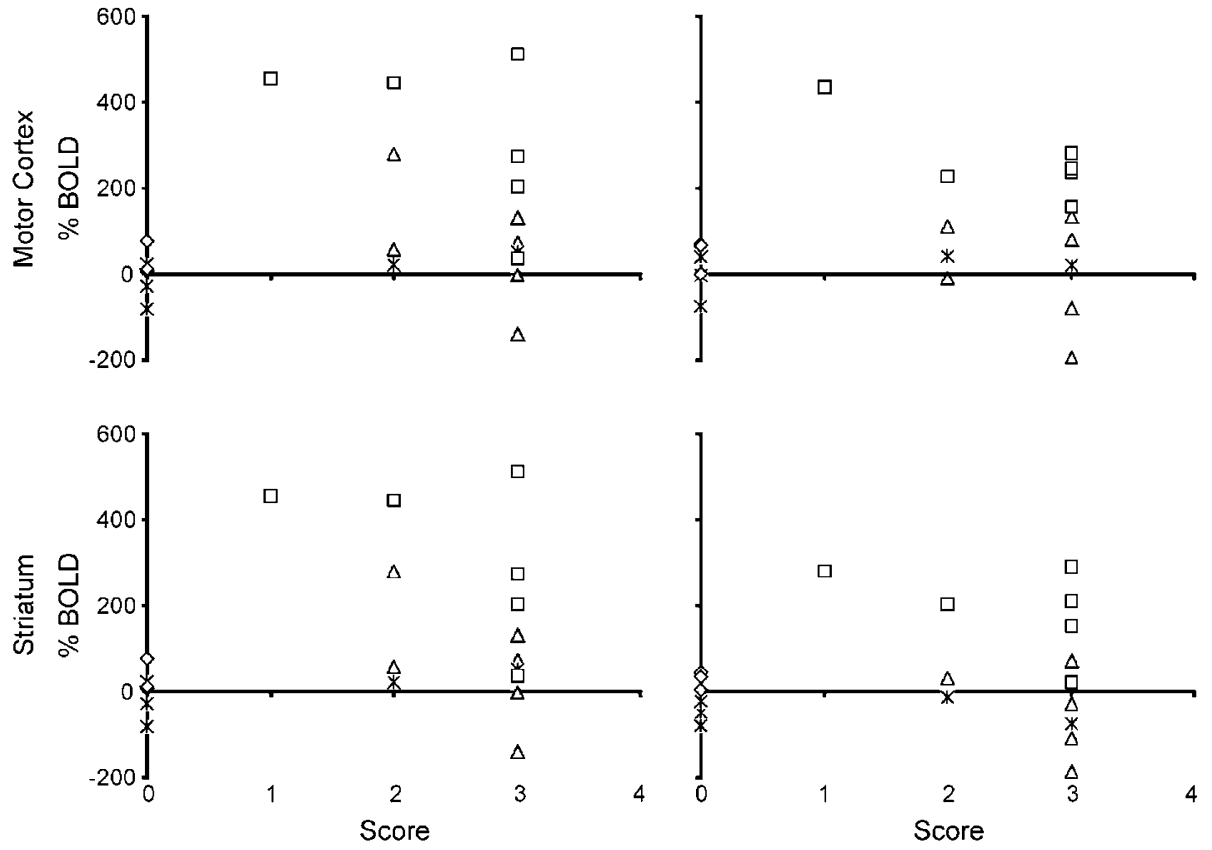

Forelimb Dyskinesia
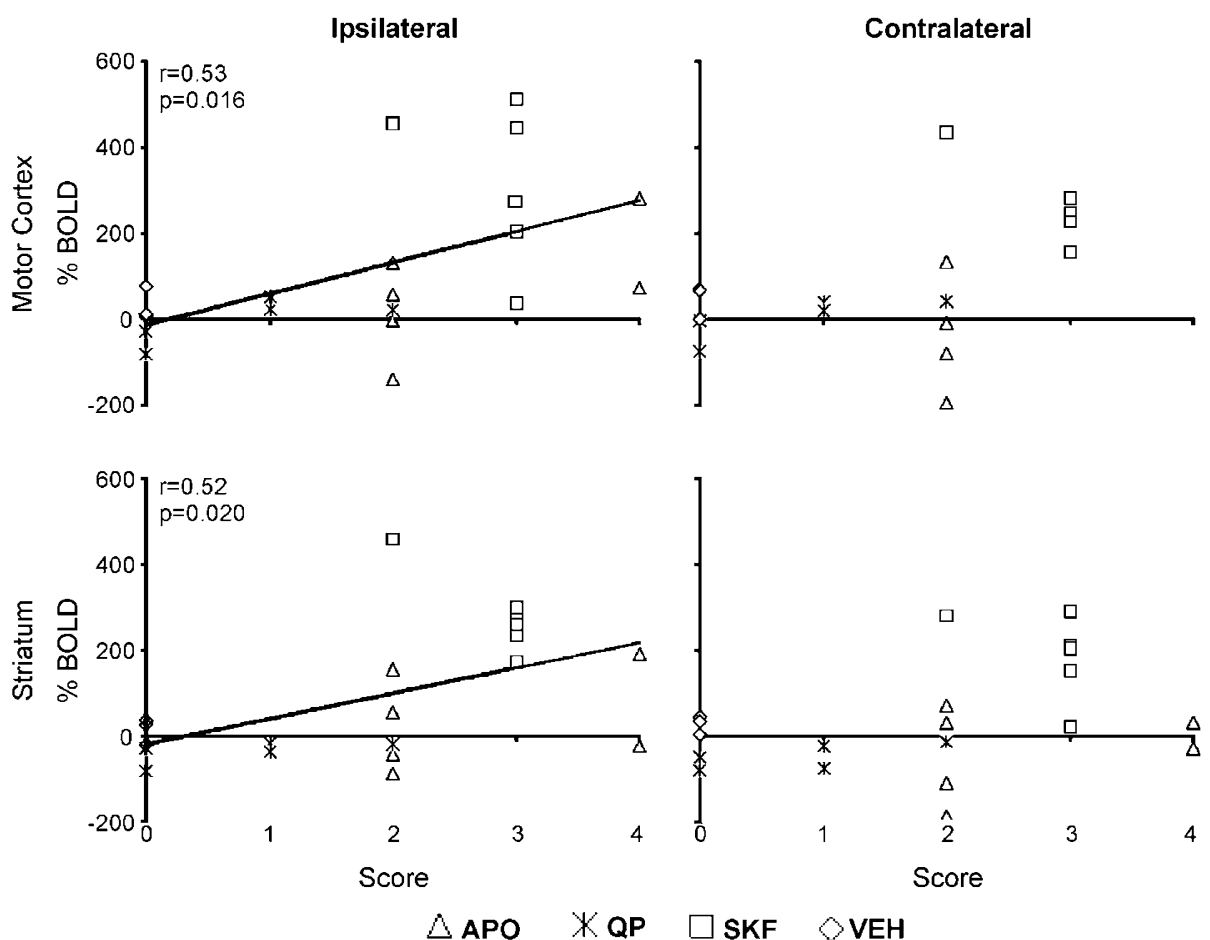

Figure 5 Correlations between scores for AIMs and BOLD signal changes in the striatum and motor cortex of 6-OHDA-lesioned rats. Significant direct correlations were found between striatal and motor cortex activation in the denervated side and dyskinesia of contralateral forelimb.

reports showing that D1/D5 agonists increase oral movements in rats and monkeys with intact dopaminergic pathways (Bedard and Boucher, 1989; Waddington et al, 1995) and produce abnormal dyskinetic oral movements in MPTP-lesioned marmosets (Gnanalingham et al, 1995). Overall, these findings are well in line with recent research showing altered D1 receptor signaling in the striatum of parkinsonian rats (Gerfen et al, 2002) and MPTP monkeys rendered dyskinetic by chronic levodopa administration (Aubert et al, 2005), and further support the usefulness of rodent models of PD for the study of dopamine agonistinduced dyskinesia. 
Pharmacological Stimulation of D1/D5 Dopamine Receptors Increases BOLD Contrast in the Denervated Striatum and Motor Cortex

There are reports regarding dopamine agonists' effects on striatal regional cerebral blood volume (rCBV) and BOLD in 6-OHDA-lesioned rats. Nguyen et al (2000) reported that systemic apomorphine produces a significant $\mathrm{rCBV}$ increase in the striatum ipsilateral to a 6-OHDA-induced lesion. Our present findings show that apomorphine induces significant asymmetric striatal activation in severely lesioned rats, which have been sensitized by prior repeated administration of apomorphine, but almost no detectable changes in rats, which have been free of dopamine agonists for 4 weeks before MRI testing. This finding is in line with results by Kalisch et al (2005) showing that apomorphine induced an asymmetric striatal BOLD response in 6-OHDA-lesioned rats, which were 'primed' with an apomorphine challenge $48 \mathrm{~h}$ before MRI. It must be noticed that in the experiments of Nguyen et al (2000) 6-OHDA-lesioned rats were repeatedly tested for apomorphine- and amphetamineinduced turning behavior before being examined and that the deployed contrast-enhanced method detects rCBV rather than endogenous BOLD changes and is more sensitive. Further studies are necessary to determine the neurovascular effects of dopamine agonists in drugnaïve parkinsonian rats, yet, it seems possible that the endogenous BOLD technique could not be sensitive enough to detect them.

The effects of selective dopamine agonists in striatal and cortical BOLD in 6-OHDA-lesioned rats have not been reported before. Here, they were assessed while maintaining constant BP with the phenylephrine BP clamping technique (Kalisch et al, 2005), thus avoiding BP-related BOLD artifacts, which can mask or simulate neuronally mediated drug effects (Kalisch et al, 2001). In normal rats, D2 family agonists increase BOLD in brain regions enriched in D3 receptors, like the nucleus accumbens, but have little if any effect in dorsal striatum and motor cortex (Ireland et al, 2005). Our present findings showing that quinpirole does not induce BOLD changes in 6-OHDA-lesioned rats are consistent with previous studies establishing that D2 family agonists do not increase 2-deoxyglucose uptake and immediate early gene expression in the striatum of parkinsonian rats (Trugman and Wooten, 1987; Robertson et al, 1989; Paul et al, 1992; LaHoste et al, 1993). Contrasting with this lack of neuronal activation in the striatum, quinpirole induces mild AIMs and strong turning behavior in 6-OHDA-lesioned rats, which were sensitized to dopamine agonists (present findings and Delfino et al, 2004; see also Robertson et al, 1989; Paul et al, 1992). On the other hand, the D1/D5 agonist SKF-81297 induced a strong striatal and cortical bilateral activation, which was higher in rats repeatedly exposed to the same agonist before MRI examination, and which was significantly higher than that induced by apomorphine. Early studies of 2-deoxyglucose uptake and immediate early gene expression recognized a powerful stimulatory effect of D1/D5 agonists in parkinsonian rats (Trugman and Wooten, 1987; Robertson et al, 1989; Paul et al, 1992; LaHoste et al, 1993). More recent work emphasized the bilateral effects of D1/D5 agonists on gene expression in the parkinsonian rat brain (Keefe and
Gerfen, 1995; Blandini et al, 2003; Taymans et al, 2005) and demonstrated that direct stimulation of D1/D5 receptors in the denervated striatum can activate the motor cortex bilaterally (Keefe and Gerfen, 1995; Blandini et al, 2003).

Altogether, our data indicate that fMRI can accurately reveal the different patterns of brain activation induced by selective dopamine agonists in parkinsonian rats, which have been repeatedly exposed to dopamine agonists before testing. Our results further indicate that the strong striatal BOLD changes induced by unselective dopamine agonists in parkinsonian rats are probably due to stimulation of D1/D5 receptors and establish that repeated administration of dopamine agonists having an action on D1/D5 receptors sensitizes striatal BOLD responsiveness to a subsequent challenge with these same drugs.

\section{Severity of FD is Related to Striatal and Motor Cortex BOLD Over-Response to Dopamine Receptor Agonists}

This is the first study trying to correlate patterns of brain activation and severity of AIMs induced by dopamine agonists in parkinsonian rats. The severity of dyskinesia in the forelimb contralateral to the lesion was linearly correlated to BOLD response in the denervated striatum and motor cortex. In contrast, no correlation was found for AD. This at first glance incongruous observation could be accounted for by different brain substrates underlying different AIMs, because in contrast to forelimb movements, trunk movements are barely represented in the motor cortex (Donoghue and Wise, 1982; Neafsey et al, 1986), and indeed, it is believed that projections to the brain stem rather than to thalamo-cortical circuits are involved in basal ganglia control of locomotion and posture (Grillner et al, 2005).

Current models of basal ganglia dysfunction predict that dopamine-agonist induced dyskinesia results from D1 receptor-mediated over-activation of striatal neurons projecting to the basal ganglia output nuclei (direct pathway) plus D2 receptor-mediated inhibition of striato-pallidal neurons (indirect pathway) (reviewed by Obeso et al, 2000; and Bezard et al, 2001). These changes would release thalamo-cortical and brain stem circuits from tonic inhibition exerted by the basal ganglia output nuclei (Filion et al, 1991; Mitchell et al, 1992). Our findings partially fit within this scheme. D2 family agonist inhibition of striatal activity could have escaped from MRI detection, and unselective dopamine agonists might have produced less striatal activation than D1/D5 agonists because of their action on D2 family receptors. In addition, dyskinesia involving the forelimb should be expected to involve contralateral motor cortex over-activation. But the neural mechanisms of dopamine agonist-induced dyskinesia are thought to essentially concern the indirect pathway (Crossman, 1990; Obeso et al, 2000) and our findings suggest a primary involvement of the D1 receptor regulated direct pathway. A primary involvement of D1 receptors and the direct basal ganglia pathway is suggested by other recent studies too (Aubert et al, 2005; Fiorentini et al, 2006), and by the fact that D2 family agonists are less likely to induce dyskinesia in patients and animal models of PD than unselective and D1/D5 selective agonists (present findings; Gnanalingham et al, 1995; Blanchet et al, 1996; Pearce et al, 
1999; Goulet and Madras, 2000; Nutt, 2000; Rascol et al, 2001; Lundblad et al, 2002; Monville et al, 2005). A recent study established that levodopa-induced dyskinesia in the rat 6-OHDA model is related to increased synchronization of afferent activity to the basal ganglia output nuclei (Meissner et al, 2006). Our present findings suggest that D1 receptor mediated over-activation of a sensitized direct pathway may contribute to driving this abnormal low frequency synchronization in the basal ganglia output nuclei and levodopa-induced dyskinesia.

Remarkably, in patients with peak-dose dyskinesia, acute levodopa has been reported to induced over-activation of the motor cortex during execution of voluntary hand movements (Rascol et al, 1998) and of the basal ganglia and motor cortex at rest (Brooks et al, 2000). Indeed, the severity of involuntary arm and hand movements correlates with increases of regional blood flow measured with PET in the contralateral motor cortex and basal ganglia (Brooks et al, 2000). Importantly, levodopa-induced over-activation of the motor cortex was not observed in drug-naïve hemiparkinsonian patients (Buhmann et al, 2003). Overall, these findings suggest that the effects of repeated administration of dopamine agonists on BOLD responses and the correlations between behavior and brain activation reported here could be related to the mechanism of levodopainduced peak-dose dyskinesia in PD.

\section{ACKNOWLEDGEMENTS}

This study was supported by DAAD and Argentine Agency for the Promotion of Science (PROALAR99-66 and PICT05-11063), National Parkinson Foundation (USA), Argentine National Research Council (CONICET) and University of Buenos Aires. We thank Dr Valeria Della Maggiore for helpful comments on an earlier version of this paper.

\section{REFERENCES}

Aubert I, Guigoni C, Hakansson K, Li Q, Dovero S, Barthe N et al (2005). Increased D1 dopamine receptor signaling in levodopainduced dyskinesia. Ann Neurol 57: 17-26.

Bedard PJ, Boucher R (1989). Effect of D1 receptor stimulation in normal and MPTP monkeys. Neurosci Lett 104: 223-228.

Bezard E, Brotchie JM, Gross CE (2001). Pathophysiology of levodopa-induced dyskinesia: potential for new therapies. Nat Rev Neurosci 2: 577-588.

Blanchet PJ, Grondin R, Bedard PJ (1996). Dyskinesia and wearingoff following dopamine D1 agonist treatment in drug-naive 1-methyl-4-phenyl-1,2,3,6-tetrahydropyridine-lesioned primates. Movement Disord 11: 91-94.

Blandini F, Fancellu R, Orzi F, Conti G, Greco R, Tassorelli C et al (2003). Selective stimulation of striatal dopamine receptors of the D1- or D2-class causes opposite changes of fos expression in the rat cerebral cortex. Eur J Neurosci 17: 763-770.

Brooks DJ, Piccini P, Turjanski N, Samuel M (2000). Neuroimaging of dyskinesia. Ann Neurol 47(Suppl 1): S154-S158; discussion S158-S159.

Buhmann C, Glauche V, Sturenburg HJ, Oechsner M, Weiller C, Buchel C (2003). Pharmacologically modulated fMRI-cortical responsiveness to levodopa in drug-naive hemiparkinsonian patients. Brain 126: 451-461.

Cenci MA, Lee CS, Björklund A (1998). -DOPA-induced dyskinesia in the rat is associated with striatal overexpression of prodynorphin- and glutamic acid decarboxylase mRNA. Eur J Neurosci 10: 2694-2706.

Cenci MA, Whishaw IQ, Schallert T (2002). Animal models of neurological deficits: how relevant is the rat? Nat Rev Neurosci 3: 574-579.

Chen Q, Andersen GK, Zhang Z, Ovadia A, Cass WA, Gash DM et al (1999). Functional MRI of basal ganglia responsiveness to levodopa in parkinsonian rhesus monkeys. Exp Neurol 158: 63-75.

Chen YCI, Galpern WR, Brownell AL, Matthews RT, Bogdanov M, Isacson O et al (1997). Detection of dopaminergic neurotransmitter activity using pharmacologic MRI: correlation with PET, microdyalisis and behavioral data. Magnet Reson Med 38: 389-398.

Crossman AR (1990). A hypothesis on the pathophysiological mechanisms that underlie levodopa- or dopamine agonistinduced dyskinesia in Parkinson's disease: implications for future strategies in treatment. Movement Disord 5: 100-108.

Delfino MA, Stefano AV, Ferrario JE, Taravini IR, Murer MG, Gershanik OS (2004). Behavioral sensitization to different dopamine agonists in a parkinsonian rodent model of druginduced dyskinesias. Behav Brain Res 152: 297-306.

Donoghue JP, Wise SP (1982). The motor cortex of the rat: cytoarchitecture and microstimulation mapping. J Comp Neurol 212: 76-88.

Filion M, Tremblay L, Bedard PJ (1991). Effects of dopamine agonists on the spontaneous activity of globus pallidus neurons in monkeys with MPTP-induced parkinsonism. Brain Res 547: 152-161.

Fiorentini C, Rizzetti MC, Busi C, Bontempi S, Collo G, Spano P et al (2006). Loss of synaptic D1 dopamine/N-methyl-D-aspartate glutamate receptor complexes in L-DOPA-induced dyskinesia in the rat. Mol Pharmacol 69: 805-812.

Gerfen CR, Miyachi S, Paletzki R, Brown P (2002). D1 dopamine receptor supersensitivity in the dopamine-depleted striatum results from a switch in the regulation of ERK1/2/MAP kinase. J Neurosci 22: 5042-5054.

Gnanalingham KK, Erol DD, Hunter AJ, Smith LA, Jenner P, Marsden CD (1995). Differential anti-parkinsonian effects of benzazepine $\mathrm{D} 1$ dopamine agonists with varying efficacies in the MPTP-treated common marmoset. Psychopharmacology (Berlin) 117: 275-286.

Goulet M, Madras BK (2000). D(1) dopamine receptor agonists are more effective in alleviating advanced than mild parkinsonism in 1-methyl-4-phenyl-1,2,3,6-tetrahydropyridine-treated monkeys. J Pharmacol Exp Ther 292: 714-724.

Grillner S, Hellgren J, Menard A, Saitoh K, Wikstrom MA (2005). Mechanisms for selection of basic motor programs - roles for the striatum and pallidum. Trends Neurosci 28: 364-370.

Ireland MD, Lowe AS, Reavill C, James MF, Leslie RA, Williams SC (2005). Mapping the effects of the selective dopamine D2/D3 receptor agonist quinelorane using pharmacological magnetic resonance imaging. Neuroscience 133: 315-326.

Kalisch R, Delfino M, Murer MG, Auer DP (2005). The phenylephrine blood pressure clamp in pharmacologic magnetic resonance imaging: reduction of systemic confounds and improved detectability of drug-induced BOLD signal changes. Psychopharmacology (Berlin) 180: 774-780.

Kalisch R, Elbel GK, Gossl C, Czisch M, Auer DP (2001). Blood pressure changes induced by arterial blood withdrawal influence BOLD signal in anesthesized rats at 7 Tesla: implications for pharmacologic MRI. Neuroimage 14: 891-898.

Keefe KA, Gerfen CR (1995). D1-D2 dopamine receptor synergy in striatum: effects of intrastriatal infusions of dopamine agonists and antagonists on immediate early gene expression. Neuroscience 66: 903-913.

LaHoste GJ, Yu J, Marshall JF (1993). Striatal Fos expression is indicative of dopamine D1/D2 synergism and receptor supersensitivity. Proc Natl Acad Sci USA 90: 7451-7455. 
Lundblad M, Andersson M, Winkler C, Kirik D, Wierup N, Cenci MA (2002). Pharmacological validation of behavioral measures of akinesia and dyskinesia in a rat model of Parkinson's disease. Eur J Neurosci 151: 120-132.

Meissner W, Ravenscroft P, Reese R, Harnack D, Morgenstern R, Kupsch A et al (2006). Increased slow oscillatory activity in substantia nigra pars reticulata triggers abnormal involuntary movements in the 6-OHDA-lesioned rat in the presence of excessive extracellular striatal dopamine. Neurobiol Dis 22: 586-598.

Metz GA, Whishaw IQ (2002). Drug-induced rotation intensity in unilateral dopamine-depleted rats is not correlated with end point or qualitative measures of forelimb or hindlimb motor performance. Neuroscience 111: 325-336.

Mitchell IJ, Boyce S, Sambrook MA, Crossman AR (1992). A 2deoxyglucose study of the effects of dopamine agonists on the parkinsonian primate brain. Implications for the neural mechanisms that mediate dopamine agonist-induced dyskinesia. Brain 115: 809-824.

Monville C, Torres EM, Dunnett SB (2005). Validation of the 1-dopa-induced dyskinesia in the 6-OHDA model and evaluation of the effects of selective dopamine receptor agonists and antagonists. Brain Res Bull 68: 16-23.

Morelli M, Fenu S, Garau L, Di Chiara G (1989). Time and dose dependence of the priming of the expression of dopamine receptor supersensitivity. Eur J Pharmacol 162: 329-335.

Murer MG, Dziewczapolski G, Menalled L, Garcia MC, Agid Y, Gershanik O et al (1998). Chronic levodopa is not toxic for remaining dopaminergic neurons, but instead promotes their recovery, in rats with moderate nigrostriatal lesions. Ann Neurol 43: $561-575$

Nagaoka T, Harel N, Zhao F, Wang P, Kim SG (2002). Critical threshold for arterial blood pressure for BOLD response to visual stimulation in anesthetized cats. Annual Meeting of the International Society of Magnetic Resonance in Medicine (ISMRM) Abstract 1367.

Neafsey EJ, Bold EL, Haas G, Hurley-Gius KM, Quirk G, Sievert CF et al (1986). The organization of the rat motor cortex: a microstimulation mapping study. Brain Res Rev 396: 77-96.

Nguyen TV, Brownell AL, Iris Chen YC, Livni E, Coyle JT, Rosen BR et al (2000). Detection of the effects of dopamine receptor supersensitivity using pharmacological MRI and correlations with PET. Synapse 36: 57-65.

Nutt JG (2000). Clinical pharmacology of levodopa-induced dyskinesia. Ann Neurol 47(Suppl 1): S160-S164; discussion S164-S166.

Obeso JA, Rodriguez-Oroz MC, Rodriguez M, DeLong MR, Olanow CW (2000). Pathophysiology of levodopa-induced dyskinesias in Parkinson's disease: problems with the current model. Ann Neurol 47(Suppl 1): S22-S32; discussion S32-S34.

Olsson M, Nikkhah G, Bentlage C, Bjorklund A (1995). Forelimb akinesia in the rat Parkinson model: differential effects of dopamine agonists and nigral transplants as assessed by a new stepping test. J Neurosci 15: 3863-3875.

Paul ML, Graybiel AM, David JC, Robertson HA (1992). D1-like and D2-like dopamine receptors synergistically activate rotation and c-fos expression in the dopamine-depleted striatum in a rat model of Parkinson's disease. J Neurosci 12: 3729-3742.

Paxinos G, Watson C (1986). The Rat Brain in Stereotaxic Coordinates, 2nd edn. Academic Press: Sidney.

Pearce RK, Jackson M, Britton DR, Shiosaki K, Jenner P, Marsden CD (1999). Actions of the D1 agonists A-77636 and A-86929 on locomotion and dyskinesia in MPTP-treated L-dopa-primed common marmosets. Psychopharmacology (Berlin) 142: 51-60.

Rascol O, Nutt JG, Blin O, Goetz CG, Trugman JM, Soubrouillard C et al (2001). Induction by dopamine D1 receptor agonist ABT431 of dyskinesia similar to levodopa in patients with Parkinson disease. Arch Neurol 58: 249-254.

Rascol O, Sabatini U, Brefel C, Fabre N, Rai S, Senard JM et al (1998). Cortical motor overactivation in parkinsonian patients with L-dopa-induced peak-dose dyskinesia. Brain 121: 527-533.

Robertson HA, Peterson MR, Murphy K, Robertson GS (1989). D1dopamine receptor agonists selectively activate striatal c-fos independent of rotational behaviour. Brain Res 503: 346-349.

Salin P, Dziewczapolski G, Gershanik OS, Nieoullon A, RaismanVozari R (1997). Differential regional effects of long-term L-DOPA treatment on preproenkephalin and preprotachykinin gene expression in the striatum of 6-hydroxydopamine-lesioned rat. Brain Res Mol Brain Res 47: 311-321.

Steward CA, Marsden CA, Prior MJ, Morris PG, Shah YB (2005). Methodological considerations in rat brain BOLD contrast pharmacological MRI. Psychopharmacology (Berlin) 80: 687-704.

Taymans JM, Kia HK, Groenewegen HJ, Leysen JE, Langlois X (2005). Bilateral control of brain activity by dopamine D1 receptors: evidence from induction patterns of regulator of $\mathrm{G}$ protein signaling 2 and c-fos mRNA in D1-challenged hemiparkinsonian rats. Neuroscience 134: 643-656.

Trugman JM, Wooten GF (1987). Selective D1 and D2 dopamine agonists differentially alter basal ganglia glucose utilization in rats with unilateral 6-hydroxydopamine substantia nigra lesions. J Neurosci 7: 2927-2935.

Tuor UI, McKenzie E, Tomanek B (2002). Functional magnetic resonance imaging of tonic pain and vasopressor effects in rats. Magnet Reson Imaging 20: 707-712.

Ungerstedt U (1976). 6-hydroxydopamine-induced degeneration of the nigrostriatal dopamine pathway: the turning syndrome. Pharmacol Ther 2: 37-40.

Waddington JL, Daly SA, Downes RP, Deveney AM, McCauley PG, O'Boyle KM (1995). Behavioural pharmacology of 'D-1-like' dopamine receptors: further subtyping, new pharmacological probes and interactions with 'D-2-like' receptors. Prog Neuropsychopharmacol Biol Psychiatry 19: 811-831.

Wang R, Foniok T, Wamsteeker JI, Qiao M, Tomanek B, Vivanco RA et al (2006). Transient blood pressure changes affect the functional magnetic resonance imaging detection of cerebral activation. Neuroimage 31: 1-11.

Winkler C, Kirik D, Bjorklund A, Cenci MA (2002). L-DOPA-induced dyskinesia in the intrastriatal 6-hydroxydopamine model of Parkinson's disease: relation to motor and cellular parameters of nigrostriatal function. Neurobiol Dis 10: 165-186. 